\title{
Neuroretinitis with Branch Retinal Artery Occlusion in a 15-Year-Old Female
}

\author{
Sina Ahmadi ${ }^{a, c} \quad$ Behrooz Azizi $^{c} \quad$ Adrian C. Tsang ${ }^{a, b} \quad$ Stuart Coupland ${ }^{a, c}$ \\ Chloe Gottlieb ${ }^{a-c}$ David Zackon ${ }^{c}$ \\ ${ }^{a}$ The Ottawa Hospital Research Institute, ${ }^{b}$ Faculty of Medicine, University of Ottawa, and \\ 'University of Ottawa Eye Institute, Ottawa, Ont., Canada
}

\section{Key Words}

Bartonella henselae $\cdot$ Neuroretinitis - Branch retinal artery occlusion - Uveitis · Cat scratch disease

\begin{abstract}
We report a case of Bartonella henselae neuroretinitis with significant disc and peripapillary edema, branch retinal artery occlusion without macula involvement and well preserved central vision. A 15-year-old female presented with loss of vision over 4 weeks in the left eye. She had a history of cat exposure, but a cat scratch, insect bite or conjunctivitis was not reported. An inferotemporal arcuate scotoma developed during the acute phase and persisted over the course of the follow-up.

(c) 2013 S. Karger AG, Basel
\end{abstract}

\section{Introduction}

Bartonella henselae infection is relatively common, and ocular involvement is seen in 5$10 \%$ of the infected patients. Ocular bartonellosis should always be considered in children and adolescents presenting with conjunctivitis, neuroretinitis or focal retinochoroiditis regardless of a history of cat or flea exposure [1]. Contamination of skin wounds or mucus membranes with flea feces and inhalation of infected material are alternative modes of transmission [2]. Infection through conjunctiva often presents as Parinaud's oculoglandular syndrome, which consists of a follicular, granulomatous conjunctivitis with associated regional tender lymphadenopathy and fever $[3,4]$. Another ocular manifestation is neuroretinitis, with mild to moderate vision loss during the acute phase, which follows a benign, self- 
Ahmadi et al:: Neuroretinitis with Branch Retinal Artery Occlusion in a 15-Year-Old Female

limiting course. Vascular occlusion is an atypical feature, but immune-compromised patients are at an increased risk $[3,4]$.

\section{Case Report}

A 15-year-old female presented with a 4-week history of progressive, painless loss of vision and inferior visual field defect in the left eye (OS). Her history was negative for red eye and discharge, but she reported having a fever and sore throat without other constitutional symptoms. Physical exam did not demonstrate other neurological disturbances or lymphadenopathy. The patient was never sexually active and her past medical history was insignificant. She reported having traveled to Vietnam for 6 weeks within the past year. The patient had contact with a friend's cat, but denied being scratched. Blood pressure at presentation was $110 / 65 \mathrm{~mm} \mathrm{Hg}$ with no history of systemic hypertension. The best-corrected visual acuity was 20/20 in the right eye (OD) and 20/40 OS. Pupils were round and reactive with a moderate left afferent pupillary defect. Color vision was also impaired OS, and a confrontation visual field test revealed an inferior field defect OS.

The anterior chamber and vitreous showed trace cells, and her intraocular pressure was $14 \mathrm{~mm} \mathrm{Hg}$ in both eyes at presentation. The posterior segment exam was normal OD, but significant disc and retinal edema, peripapillary serous retinal detachment and vascular congestion were observed OS. A characteristic partial macular star sparing the area temporal to the fovea was also observed OS (fig. 1). Subfoveal fluid was present and confirmed by optical coherence tomography. A branch of the superior-temporal retinal artery was occluded, but the arteriole supplying the superior part of the macula remained patent (fig. 1, fig. 2). Venous tortuosity and peripapillary telangiectasia were present, but there was no sign of concomitant vein occlusion (fig. 1, fig. 2). A clinical diagnosis of $B$. henselae vaso-occlusive neuroretinitis was made, and doxycycline $100 \mathrm{mg}$ p.o. b.i.d. was prescribed. The $B$. henselae IgG titer was elevated (1:128). Lyme and syphilis serologies and a purified protein derivative skin test were negative. While not routine in cases with a high clinical suspicion of $B$. henselae infection, an MRI of the brain and orbits as well as a chest Xray were performed to exclude other infectious etiologies.

After 2 months of doxycycline therapy, the disc and retinal edema had improved significantly and most retinal vessels returned to normal caliber (fig. 2). Visual acuity improved to 20/20 with improved color vision, but the left afferent pupillary defect was still present. Central visual field defects improved with the resolution of macular edema, but the branch retinal artery occlusion resulted in inner retinal infarct with a persistent inferior arcuate scotoma (fig. 3).

\section{Discussion}

This was a case of $B$. henselae neuroretinitis with occlusive vasculitis in an immunocompetent patient with persistent visual field defect and an absent history for cat scratch disease or Parinaud's oculoglandular syndrome. The diagnosis was not made based on an elevated $B$. henselae titer alone, but in the context of a strong clinical suspicion of $B$. henselae infection and an exclusion of other common infectious etiologies. B. henselae-associated neuroretinitis typically follows a benign and self-limited course, but the potential for significant visual complications cannot be overlooked. Previous studies have reported vascular occlusive events in as high as $17-21 \%$ of patients with posterior segment manifestations of a $B$. 
Ahmadi et al.: Neuroretinitis with Branch Retinal Artery Occlusion in a 15-Year-Old Female

henselae infection $[5,6]$. The efficacy of antibiotic regimens in patients with $B$. henselae neuroretinitis has not been proven with controlled studies [7]. B. henselae should be considered in adolescents or young adults with vascular occlusion, optic disc edema and macular fluid. An elevated $B$. henselae titer is supportive of a diagnosis despite a negative history of cat scratch or bite.

\section{References}

1 Cunningham ET, Koehler JE: Ocular bartonellosis. Am J Ophthalmol 2000;130:340-349.

-2 Chomel BB, Boulouis HJ, Maruyama S, Breitschwerdt EB: Bartonella spp. in pets and effect on human health. Emerg Infect Dis 2006;12:389-394.

-3 Jacobs RF, Schutze GE: Bartonella henselae as a cause of prolonged fever and fever of unknown origin in children. Clin Infect Dis 1998;26:80-84.

-4 Roe RH, Jumper JM, Fu AD, Johnson RN, McDonald HR, Cunningham ET: Ocular Bartonella infections. Int Ophthalmol Clin 2008;48:93-105.

5 Ormerod LD, Skolnick KA, Menosky MM, Pavan PR, Pon DM: Retinal and choroidal manifestations of catscratch disease. Ophthalmology 1998;105:1024-1031.

-6 Solley WA, Martin DF, Newman NJ, King R, Callanan DG, Zacchei T, Wallace RT, Parks DJ, Bridges W, Sternberg P: Cat scratch disease: posterior segment manifestations. Ophthalmology 1999;106:1546-1553.

7 Chi SL, Stinnett S, Eggenberger E, Foroozan R, Golnik K, Lee MS, Bhatti T: Clinical characteristics in 53 patients with cat scratch optic neuropathy. Ophthalmology 2012;119:183-187.
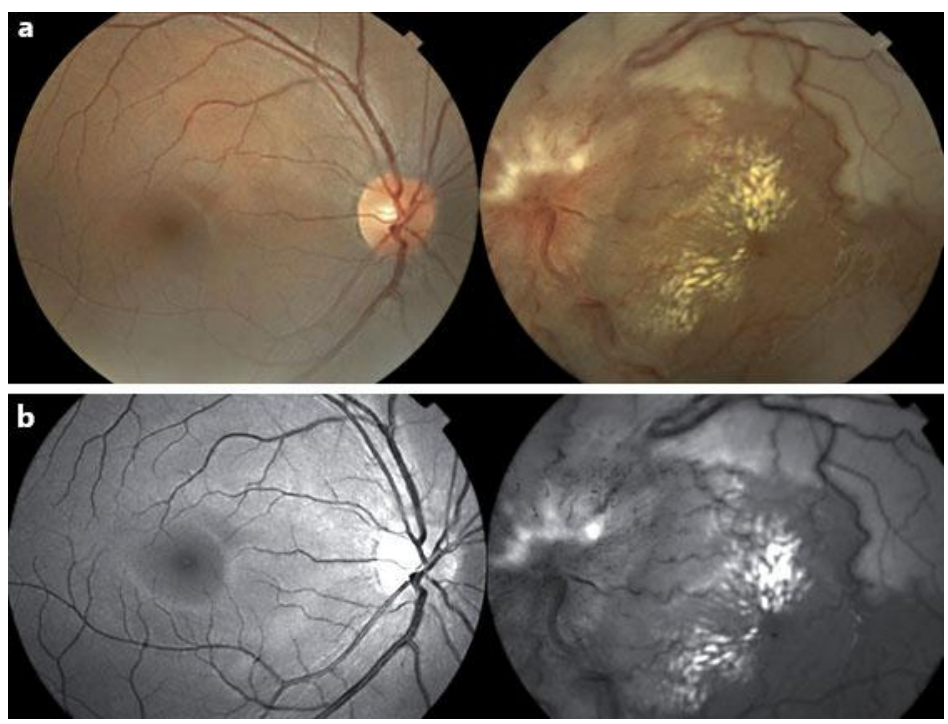

Fig. 1. Color fundus photograph (a) and red free image (b) of both eyes at presentation. The left eye shows signs of neuroretinitis and an inner retinal infarct due to a superotemporal branch retinal artery occlusion. 


\section{Case Reports in \\ Ophthalmology}

\begin{tabular}{l|l}
\hline Case Rep Ophthalmol 2013;4:265-268 \\
\hline DOI: 10.1159/000356935 & $\begin{array}{l}\text { @ 2013 S. Karger AG, Basel } \\
\text { www.karger.com/cop }\end{array}$ \\
\hline
\end{tabular}

Ahmadi et al.: Neuroretinitis with Branch Retinal Artery Occlusion in a 15-Year-Old Female

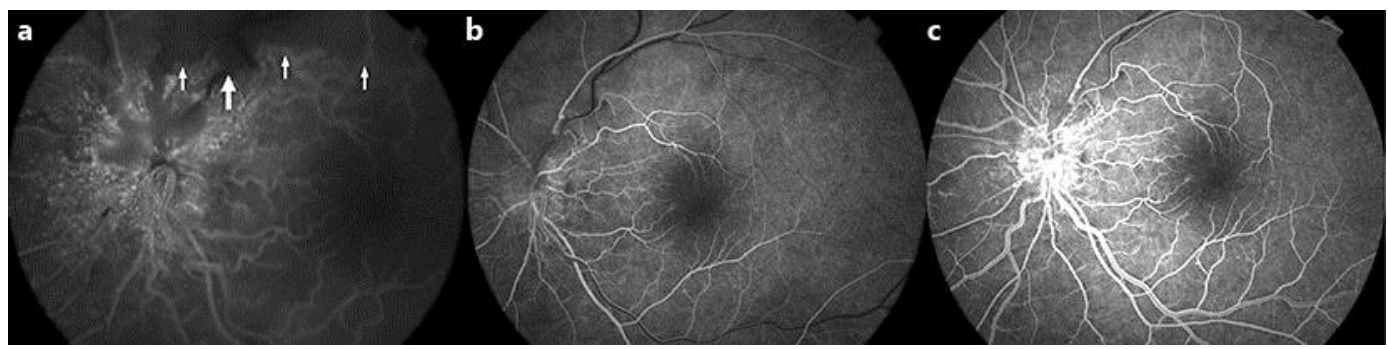

Fig. 2. a Left eye fluorescein angiogram, venous phase, shows generalized vascular congestion, capillary nonperfusion in the superior vascular arcade territory (small arrows) and reduced superotemporal venous return (large arrow). Perfusion of the central macula is largely preserved. b, c Follow-up fluorescein angiogram of the left eye after 2 months. Early venous (b) and venous (c) phase. Despite reperfusion of the previously infarcted superior retina, superotemporal venous return is still significantly delayed.

a
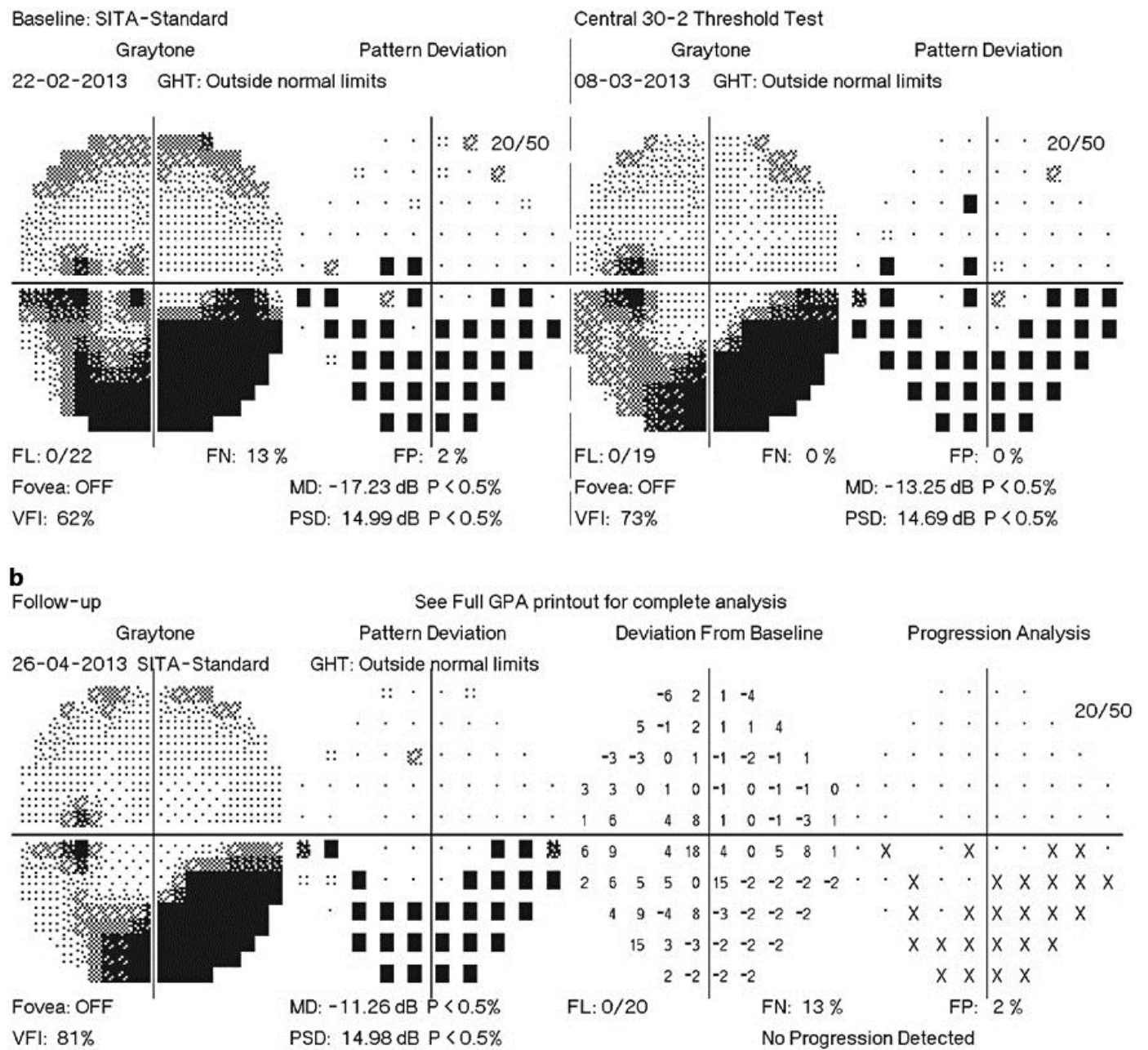

Fig. 3. Baseline Humphrey 30-2 visual fields at presentation (a) and 2 weeks later (b). Follow-up visual field at 2 months shows improvement of central defects and a diminished blind spot after the resorption of edema. Inferior arcuate scotoma persisted due to inner retinal infarct in the acute phase. 